\title{
New models of massive and Wolf-Rayet stars with mass loss and rotation
}

\author{
André Maeder \\ Geneva Observatory, CH-1290 Sauverny, Switzerland
}

\begin{abstract}
We give results of models of massive stars which have a detailed physical treatment of rotation, including structural equations for shellular rotation, new treatments of shears in differentially rotating stars and of meridional circulation, together with mass loss rates depending on rotation. For a $20 \mathrm{M}_{\odot}$ star, He- and N-enrichments at the stellar surface already occur during the MS phase for moderately low rotational velocities, thus most supergiants are enriched in helium and nitrogen. A long B- and A-supergiant phase results from rotational mixing, with some primary nitrogen formed at this stage. For the most massive stars, rotation makes the star to enter the WR stage during the MS phase thus avoiding the LBV and red supergiant stage.

The WR life-times are considerably increased by rotation and the minimum mass for forming WR stars is lowered. Interestingly enough, the increase of the WN life-time is larger than for WC stars, so that rotation leads to a decrease of the $\mathrm{WC} / \mathrm{WN}$ number ratio. Also, the fraction of transition WN/WC stars is much larger at higher rotation.

Finally, on the basis of clusters in the SMC, in the LMC and towards the galactic interior and exterior, we show that for clusters with ages between about 1 and $3 \times 10^{7} \mathrm{yr}$ the fraction of Be stars with respect to normal B stars is larger at lower metallicities. This may suggest a higher rotation at lower metallicities for massive stars, due to a different history of star formation.
\end{abstract}

\section{Global view on Wolf-Rayet models}

Let us firstly recall that the existing grids of models are in excellent agreement with a number of properties of WR stars ( $c f$. Maeder \& Conti 1994):

- the observed abundances of CNO elements in WN stars and of He, C, O and Ne in WC stars;

- the luminosities of WR stars;

- the changes of the number ratios $\mathrm{WR} / \mathrm{O}$ and $\mathrm{WC} / \mathrm{WN}$ in galaxies of various metallicities;

- the distribution of WC subtypes in galaxies, in particular why are WC9-WC8 found in the galactic interior and why only WC4-WC6 in the exterior of the Galaxy and in the LMC ( $c f$. Smith \& Maeder 1991); and

- the differences in the luminosity of WC subtypes at a given metallicity ( $c f$. Smith \& Maeder 1991).

WR stars may result from various channels ( $c f$. Maeder 1982): mass loss from O-stars, mass loss from red supergiants, binary evolution and internal mixing. The relative importance of these channels has to be appreciated properly, which is not yet the case. To show the problem, let us consider the binary channel. In 
the SMC, a large fraction, if not the totality, of WR stars are likely to be binaries and may perhaps result from binary mass transfer. However, the WR/O ratio in the SMC is only about 0.015 . In the solar neighbourhood the WR/O ratio is $\sim 0.10$ and in the galactic interior $\sim 0.20$. This means that if the binary mass transfer plays a similar role in both galaxies (a point which we do not know), about $85 \%$ of the WR stars in the solar neighbourhood owe their existence to a process other than binary mass transfer, possibly mass loss and rotation. This fraction would be higher than $90 \%$ in the galactic interior. This example illustrates how the various channels may have a different importance depending on metallicity.

There is another point I want to strongly emphasize. Stellar models can be applied (e.g., for WR stars, star populations, starbursts) only if they have been carefully tested on the basis of star clusters. In the case of the Geneva models this was done by Mermilliod \& Maeder (1986) and Meynet et al. (1993). Such tests do not seem to have been made for all models currently used.

Now, why new models with rotation? OB stars have in general fast rotation velocities. Also, evidence for He- and $\mathrm{N}$-excesses has been found in a number of cases: fast rotating O-stars (Herrero et al. 1997), B- and A-supergiants in particular in the SMC (Venn et al. 1998). A second parameter seems necessary to account for the properties of WN stars without H ( $c f$. Smith \& Maeder 1998). The explanation for the number ratio of red-to-blue supergiants also seems to require some additional mixing (Langer \& Maeder 1995). New models with rotation are now in progress in various groups, paticularly by $\mathrm{N}$. Langer and at the Geneva Observatory.

\section{Basic physics in rotating models}

Without going into the details of the physics of the rotating models, I shall briefly mention the main physical processes:

- 'Shellular' rotation: such a law of the form $\Omega=\Omega(\mathrm{r})$ has been proposed by Zahn (1992), as a result of horizontal turbulence, which limits the degree of horizontal differential rotation.

- Basic equations of structure: the classical scheme of equations has been rewritten for shellular rotation (Meynet \& Maeder 1997).

- Shears are the main instability sources. We take as an hypothesis that the excess of energy in the shear is injected in the spectrum of turbulence at the thermal timescale. This is leading to a new diffusion coefficient (Maeder 1997), identical to that by Zahn (1992) in case of very high rotation and to that for semiconvection by Langer et al. (1983) in the case of no rotation.

- Meridional circulation is accounted for with the recent improvements by Zahn (1992) and Maeder \& Zahn (1998). This provides a new expression which properly includes the reduction of the velocity of meridional circulation due to the $\mu$-gradients, while before only the limiting velocity permitted by the $\mu$-gradient was considered.

- Surface effects: the global increases of the mass loss rates due to rotation are accounted for ( $c f$. Friend \& Abbott 1986). Further developments will allow us to account for the anisotropic mass loss over the stellar surface, which selectively removes the angular momentum (Maeder 1999). 
- Evolution of $\Omega$ and abundances is followed-up with the above processes being taken into account by the appropriate equation for the transport and conservation of angular momentum.

\section{Model results}

\subsection{Evolution of $\Omega$}

Detailed calculations show that the $\Omega$-profile in a rotating star rapidly reaches an equilibrium, in a time $<3 \times 10^{4} \mathrm{yr}$ at $20 \mathrm{M}_{\odot}$, as a result of a compensation between the outwards transport of angular momentum by shears and the inwards transport by meridional circulation. After the equilibrium is reached and at least during the rest of the MS phase, the internal $\Omega$-profile is mainly determined by the local conservation of angular momentum during contraction and expansion phases. $\Omega(\mathrm{r})$ is a smoothly declining function of $\mathrm{r}$, with a higher degree of differential rotation for the initially faster rotating stars.

\subsection{Internal $H$-distribution and surface $N / C$ excess}

The distribution of hydrogen in a $20 \mathrm{M}_{\odot}$ star with rotation is shown in Figures 1a and $1 \mathrm{~b}$. We note that the height of the $\mu$-discontinuity at the edge of the convective core is very much reduced by rotation. Also, there is a mild $\mu$ gradient built up in external regions.

We notice that for $\Omega / \Omega_{c}=0.4$ the convective core shrinks during MS evolution while it increases for $\Omega / \Omega_{c}=0.6$. This behaviour, i.e., the reduction or the growth of the convective core, determines respectively the redwards and bluewards evolutionary tracks in the HR diagram during the MS phase.
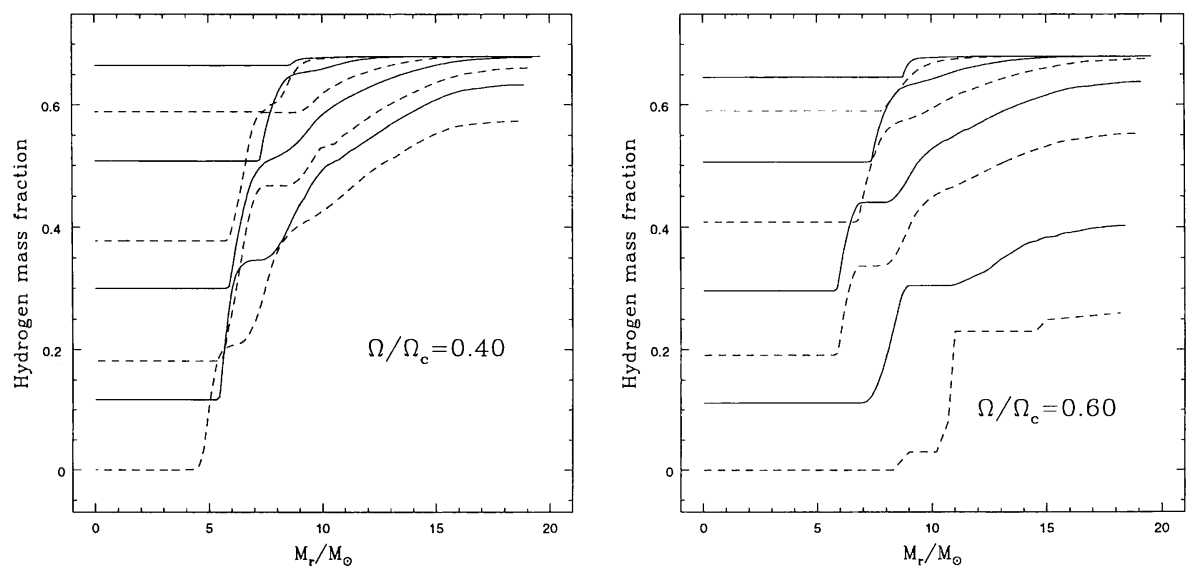

Figure 1. Internal $\mathrm{H}$-profiles in a $20 \mathrm{M}_{\odot}$ star during $\mathrm{MS}$ evolution for two values of the initial ratio $\Omega / \Omega_{c}=0.4$ (left) and 0.6 (right). $\Omega / \Omega_{c}$ is the fraction of the angular velocity at break-up on the zero-age sequence. Remark: the case of zero rotation almost exactly corresponds to the inner envelope of the various curves for $\Omega / \Omega_{c}=0.4$ (from Meynet \& Maeder 1999). 


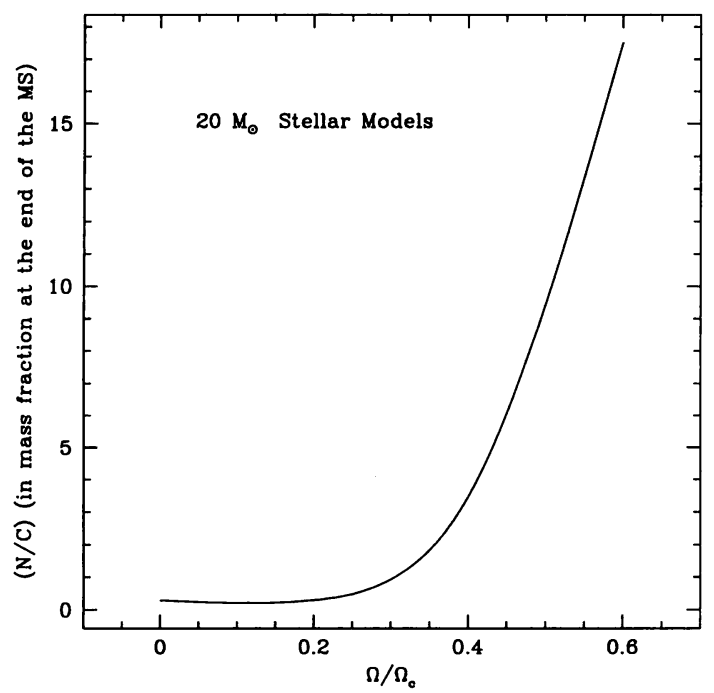

Figure 2. Increase of $\mathrm{N} / \mathrm{C}$ at the end of the MS phase with rotation for an initial $20 \mathrm{M}_{\odot}$ star. $\Omega / \Omega_{c}$ is the fraction of the angular velocity at break-up on the zero-age sequence.

To these changes of the internal H-profiles, a surface enhancement of the $\mathrm{N} / \mathrm{C}$ ratio is associated as illustrated by Figure 2 . Even a moderate rotation $\left(\Omega / \Omega_{c}>0.3\right)$ will bring $\mathrm{N}$ to the surface during the MS phase, so that a $\mathrm{N} / \mathrm{C}$ excess will be present from the blue supergiant phase onwards. Also, we may note that the effects of rotation are larger for larger initial masses.

\subsection{HR diagram with rotation}

The HR diagram of evolution with rotation shows a number of highly interesting features. Evolution is a function of mass, metallicity and rotation, the three parameters being almost of comparable importance for the massive stars! Let us briefly emphasize a few of the most significant results:

- Rotation introduces a large scatter in the mass-luminosity relation.

- At $60 \mathrm{M}_{\odot}$ even a moderate rotation $\left(\Omega / \Omega_{c} \simeq 0.3\right)$ will move the star to the WR stage during the MS, thus avoiding redwards evolution towards the LBV and red supergiant stages.

- The larger the rotation, the earlier the entry into the WR phase. As a consequence of this early entry into the WR phase the total mass loss is larger and the WR star evolves to lower masses and luminosities, but they still follow the typical mass-luminosity relation of WR stars (Maeder 1983; Schaerer \& Maeder 1992).

- For a $20 \mathrm{M}_{\odot}$ star, fast rotation may even produce an initial bluewards track.

- For intermediate rotation at $20 \mathrm{M}_{\odot}$, a long B- and A-supergiant phase occurs, with significant He- and N-enhancements. These supergiants beautifully 


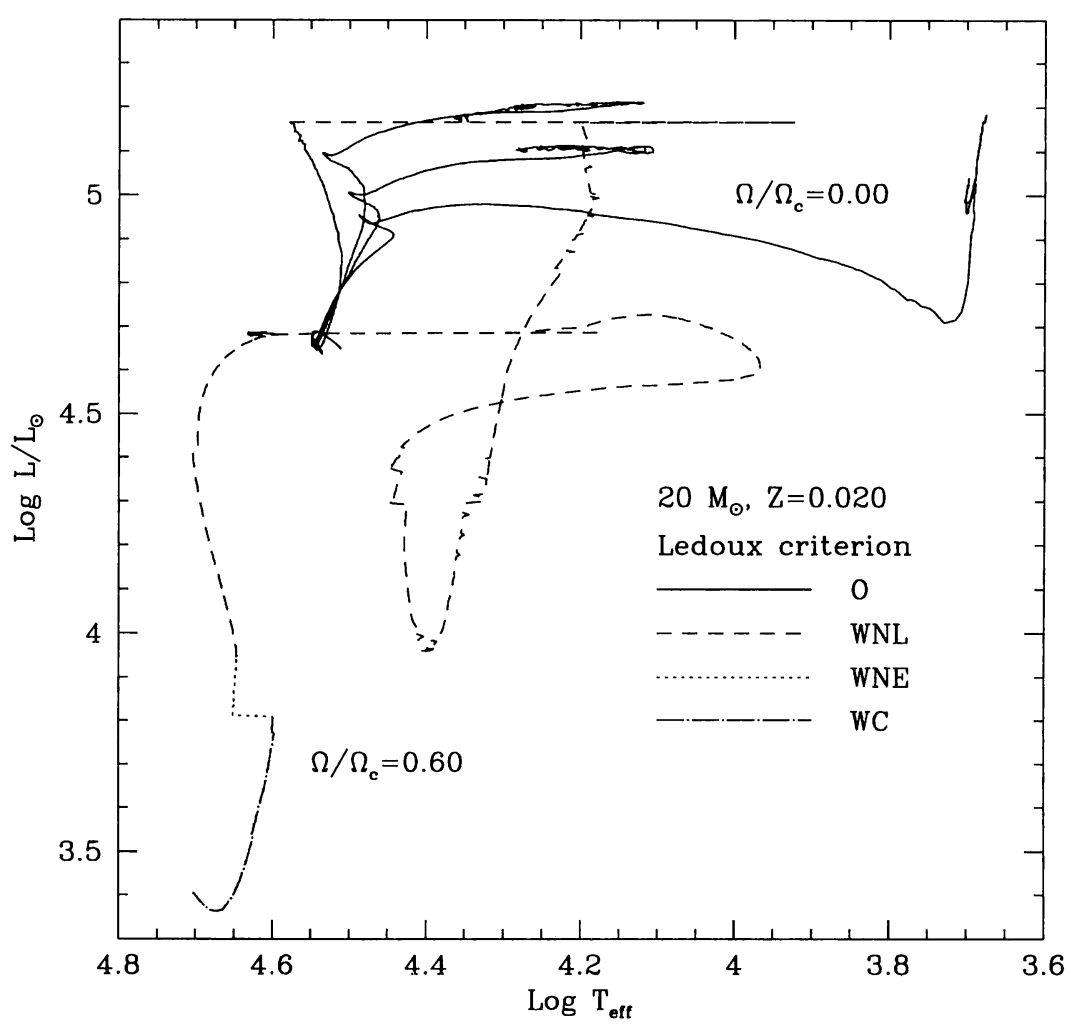

Figure 3. Evolution of a $20 \mathrm{M}_{\odot}$ star for different initial ratios $\Omega / \Omega_{c}$. The two intermediate cases with $\Omega / \Omega_{c}=0.2$ and 0.4 have been followed up until a central value $Y_{\mathrm{c}}=0.30$. Note the interesting correspondence with Fig. 1 of Fliegner \& Langer (1995).

correspond to those observed by Humphreys et al. (1991), which are in significant number, particularly in the Magellanic Clouds and with strong $\mathrm{N}$ - and He-enrichments.

Most remarkably, there is some primary nitrogen formed during the above mentioned long B- and A- supergiant phase. Such stars have both a Heburning core and a $\mathrm{H}$-burning shell and the transport by shears and meridional circulation is sufficient to bring some new ${ }^{12} \mathrm{C}$ from the core to the H-burning shell where it is immediately transformed into ${ }^{14} \mathrm{~N}$, which is thus primary nitrogen. This additional amount of $\mathrm{CNO}$ elements boosts the nuclear reaction rates in the shell whose luminosity is strongly enhanced. In turn, the convection which brings hydrogen from the outer layers to the shell favours a long duration of the enhanced shell, which may even experience some migration towards the interior. These effects give rise to the many observed B- and A-type supergiants with the remarkable $\mathrm{N}$ - and He-enhancements observed. 


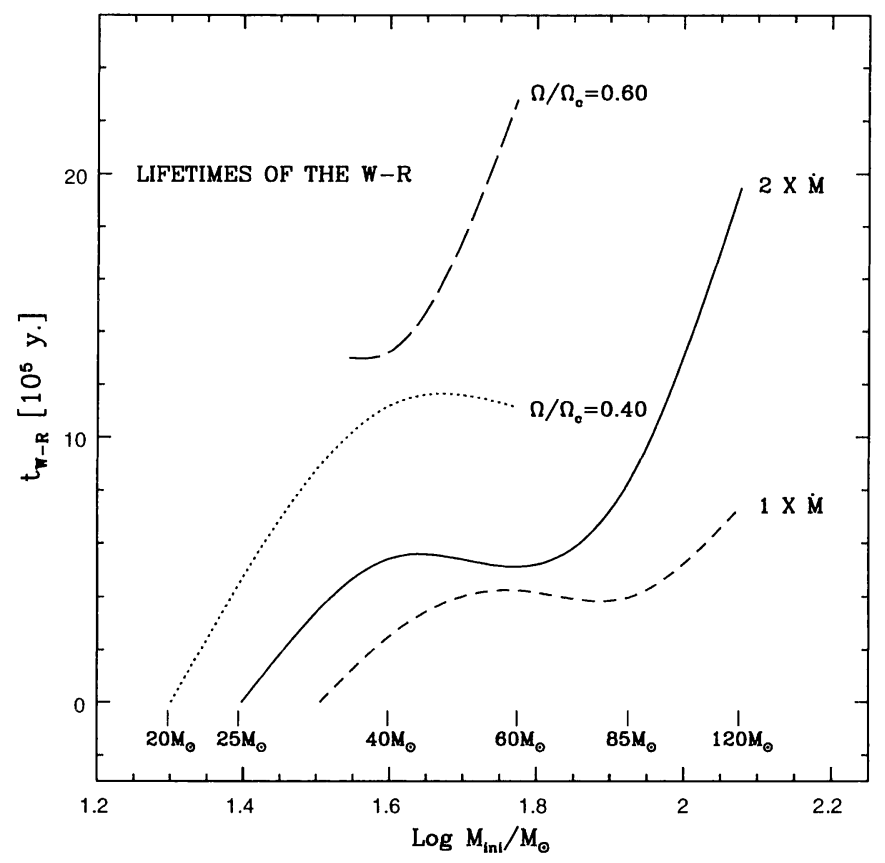

Figure 4. WR life-times for models with mass loss: $1 \times \dot{M}$ (cf. Schaller $e t$ al. 1992); $2 \times \dot{M}$ (cf. Meynet et al. 1994) and some results with rotational effects ( $c f$. Meynet \& Maeder 1999).

\subsection{Wolf-Rayet life-times}

Since mixing reduces the amount of hydrogen in the external layers, the composition typical of WR stars, say a surface H-content $X_{s}<0.4$, is reached earlier. Thus, rotation both increases the WR life-times and lowers the minimum mass for forming WR stars, in a way which does not depend very much on the exact value of the chosen limit for $X_{s}$.

Quite interestingly, rotation could also help to understand why the observed $\mathrm{WR} / \mathrm{O}$ number ratio in I Zw 18 is about 0.02 (cf. Legrand et al. 1997), whereas current models would only predict a WR/O ratio of about 0.001 at a metal content equal to $1 / 40$ of the solar composition.

New models for metal-deficient starbursts also require the knowledge of the distribution of rotational velocities, in order that simulations can be made with calculated tracks convoluted over the observed distribution of rotation velocities.

\section{5. $\quad W C / W N$ ratios and transition $W N / W C$ stars}

Another interesting effect is that rotation decreases the ratio of WC/WN lifetimes ( $c f$. Figure 5). The main reason is that mixing in rotating stars produces a wide zone with a mixture of $\mathrm{He}$ and $\mathrm{H}$, and it is only when this very extended mixed zone has been removed that the star will leave the WNL phase, thus 


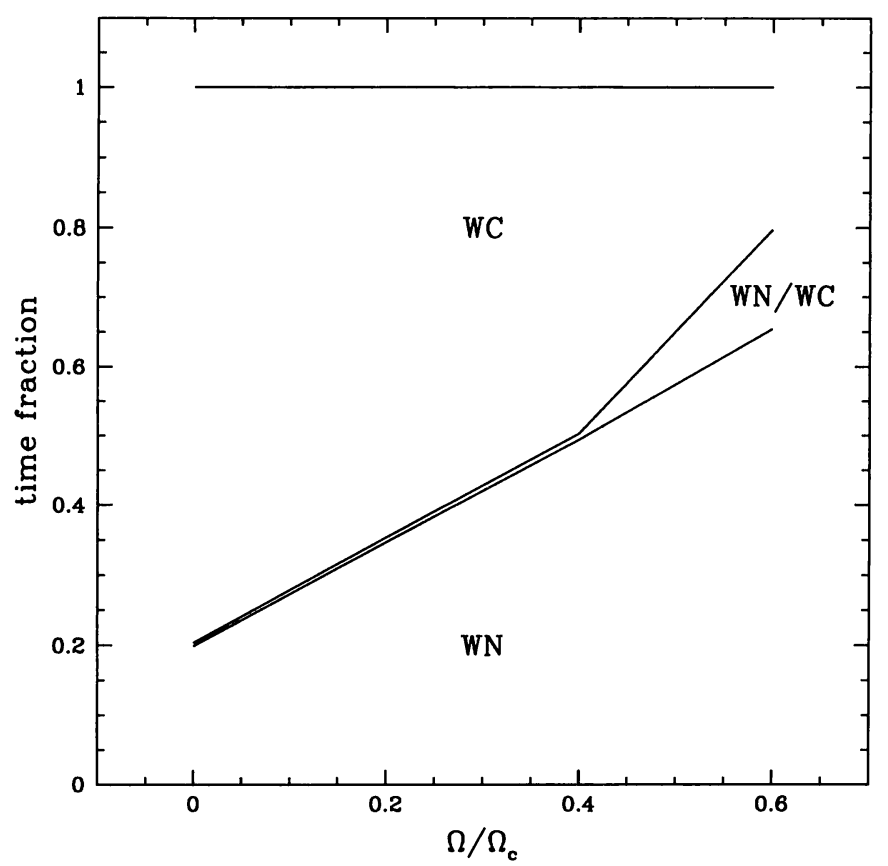

Figure 5. Change of the WC and WN life-time ratio as a function of the initial $\Omega / \Omega_{c}$ for an initial $60 \mathrm{M}_{\odot}$. Note that the total WR life-time is also growing.

leading to a reduced $\mathrm{WC} / \mathrm{WN}$ number ratio, even if both the $\mathrm{WC}$ and $\mathrm{WN}$ life-times are increased.

The WNE phase (assumed without $\mathrm{H}$ ) is short and followed by a transitory intermediate WN/WC phase, where both $\mathrm{N}$ - and C-rich layers are observed ( $c f$. Conti \& Massey 1989). As a result of the more shallow internal composition gradient the fraction of the life-time in the transitory intermediate WN/WC phase is growing with rotation, as illustrated in Figure 5.

Future studies will also compare the observed and theoretical distributions of rotational velocities for O-stars and supergiants and include the anisotropies in the losses of mass and of angular momentum.

\section{Stellar rotation in galaxies}

Are rotation velocities the same in different galaxies? We do not know yet. However, star formation is very much influenced by the radiation from grains, and thus by metallicity, therefore the above question is legitimate.

At present we have some preliminary indications from the Be phenomenon, which is clearly related to fast rotation ( $c f$. Mazzali et al. 1996). Feast (1972) was the first to notice a high fraction of Be stars in the Magellanic Clouds. 


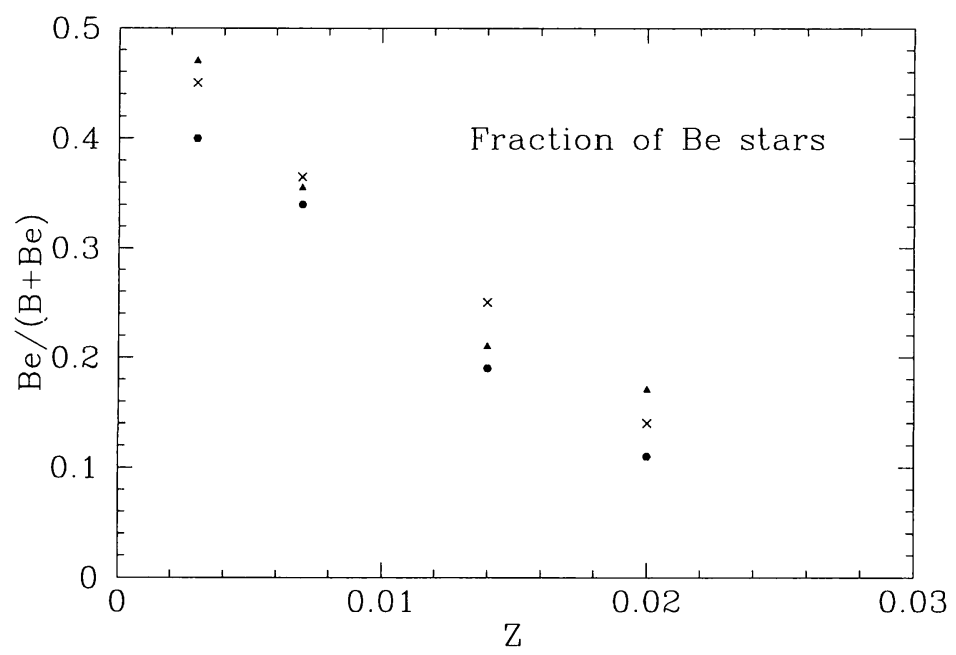

Figure 6. Relation between the fraction $\mathrm{Be} /(\mathrm{B}+\mathrm{Be})$ and the average metallicity for 16 clusters in the SMC, LMC and in the Galaxy. Magnitude intervals: $-4,-2$ (triangles); $-5,-2$ (dots); $-5,-1.4$ (crosses).

Further compelling evidences were given by Grebel et al. $(1994,1996)$ and Grebel (1997).

As shown by Mermilliod (1982), the Be/B fraction is a function of cluster ages, with a maximum for clusters with turnoff between O9.5 and B3 (i.e., ages about 1 to $3 \times 10^{7} \mathrm{yr}$ ). Thus we have to make a careful selection of clusters in the SMC, in the LMC and in the Milky Way for this range of ages in order to properly disentangle metallicity and age effects. This way, 16 clusters with more than 500 stars in the considered interval of ages have been examined and the fraction of Be stars as a function of metallicity $Z$ has been examined. The results are summarized in Figure 6, from a work in preparation with E. Grebel, J.C. Mermilliod and M. Azzopardi. Fig. 6 shows a clear increase of the number ratio $\mathrm{Be} /(\mathrm{B}+\mathrm{Be})$ for decreasing metallicities.

Another independent observation supports the view that rotation velocities might be higher in the SMC. The N/H excesses for B- and A-supergiants are quite large in the SMC (excesses by a factor of 10-20), while in the Milky Way they are moderate (excesses by a factor of 3) as shown by Venn (1998). This result clearly supports a stronger mixing in the SMC and possibly even the creation of some primary N. At this stage the above suggestion of a higher rotation in the SMC still needs further observational confirmation and related theoretical studies. If confirmed, this will have major implications on stellar evolution and nucleosynthesis at low metallicities and in the first stellar generations.

Acknowledgments. I express my best thanks to Dr. Georges Meynet for a fruitful collaboration on all the problems of stellar rotation and evolution. 


\section{References}

Conti, P.S., Massey P. 1989, ApJ 337, 251

Feast, M.W. 1972, MNRAS 159,113

Fliegner, J., Langer, N. 1995, in: K.A. van der Hucht \& P.M. Williams (eds.), WolfRayet Stars: Binaries, Colliding Winds, Evolution, Proc. IAU Symp. No. 163 (Dordrecht: Kluwer), p. 326

Friend, D., Abbott, D. 1986, ApJ 311, 701

Grebel, E.K. 1997, A\&A 317, 448

Grebel, E.K., Roberts, W.J., Will, J.M., de Boer, K.S. 1994, SSR 66, 65

Grebel, E.K., Roberts, W.J., Brandner, W. 1996, A\&A 311, 470

Herrero, A., Villmariz, M.R., Martin, E.L. 1998, in: I.D. Howarth (ed.), Boulder-Münich

II: Properties of Hot Luminous Stars, ASP-CS 131, 159

Humphreys, R.M., Kudritzki, R.P., Groth, H.W. 1991, A\&A 245, 593

Langer, N., Fricke, K.J., Sugimoto, D. 1983, A\&A 126, 207

Langer, N., Maeder, A. 1995, A\&A 295, 685

Legrand, F., Kunth, D., Roy, J.R., Mas-Hesse, J.M., Walsh, J.R. 1997, A\&A 326, L17

Maeder, A. 1982, A\&A 105, 149

Maeder, A. 1997, A\&A 321, 134 (paper II)

Maeder, A. 1999, A\&A in press

Maeder, A., Conti, P.S. 1994, ARAA 32, 227

Maeder, A., Zahn, J.P. 1998, A\&A 334, 1000 (paper III)

Mazzali, P.A., Lennon, D.J., Pasian, F., Marconi, G., Baade, D., Castellani, V. 1996, A\&A 316,173

Mermilliod, J.-C. 1982, A\&A 109, 48

Mermilliod, J.-C., Maeder, A. 1986, A\&A 158, 45

Meynet, G., Maeder, A. 1997, A\&A 321, 465

Meynet, G., Maeder, A. 1999, A\&A in preparation

Meynet, G., Maeder, A., Schaller, G., Schaerer, D., Charbonnel, C. 1994, A\&AS 103, 97

Meynet, G., Mermilliod, J.-C., Maeder, A. 1993, A\&A 98, 477

Schaller, G., Schaerer, D., Meynet, G., Maeder, A. 1992, A\&AS 96, 269

Schaerer, D., Maeder, A. 1992, A\&A 263, 129

Smith, L.F., Maeder, A. 1991, A\&A 241, 77

Smith, L.F., Maeder, A. 1998, A\&A 334, 845

Venn, K.A., McCarthy, J.K., Lennon, D.J., Kudritzki, R.P. 1998, PASP 131, 177

Zahn, J.P. 1992, A\&A 265, 115

\section{Discussion}

Vanbeveren: Comment: rotation is necessary to have the Be phenomenon but it is not sufficient. So relating rotation and the metallicity be means of the number of Be stars in low metallicity regions may be too simple. Question: we have heard during the review of A. Willis that only $15 \%$ of the single WR stars shows spectral polarimetric signatures of rotational effects in the stellar wind. Can you relate this observation to your rotating evolutionary models? 
Maeder: Sorry, but I do not see why fast rotation is not sufficient to produce the Be phenomenon. What would you need in addition? My understanding of Willis' talk is just that $15 \%$ of the WR nebulae is anisotropic. To relate this to rotation, we need to know above which rotational velocity a WR star creates an anisotropic nebula as observed. In this context, we have to keep in mind that the WR winds are very fast anyway.

Hill: The Be phenomenon may be related to pulsation which in turn might depend on metallicity, so one has to be careful interpreting the dependance of Be fraction on $Z$.

Maeder: As a matter of fact, I do not know compelling evidences that the Be phenomenon is basically a consequence of pulsations. On the contrary the average $v_{\text {rot }}$ of Be stars is higher than for normal B stars and rotation is thus certainly the leading factor.

Peimbert: Does rotation increase also the life-time of the main sequence phase of O-type stars? How is the WR/O number-ratio effected by rotation?

Maeder: The answer to both questions is yes. Rotation is increasing the MS phase, it is also increasing the WR life-times (in general much more), so that the WR/O lifetime ratios are also finally increased. Interestingly enough, rotation also increases the WN/WR life-time ratios, in a sense satisfactory in view of the observations, which were not showing as many WC stars as predicted by high mass-loss rate models.

Herrero: Is there a minimum rotational velocity for having $\mathrm{He}$ - and $\mathrm{N}$-enhancements during the MS phase?

Maeder: Yes, there is such a minimum rotation. However, the exact value depends on the stellar mass considered. In the sense that the larger the mass, the lower needs the rotational velocity to be, in order to produce some He- and $\mathrm{N}$-enhancements.

Schmutz: One of your motivation points to include rotation in the stellar models was that there is a need for a two-dimensional classification of WN stars. Now you did not mention whether you can now explain this 2-D property with rotating models.

Maeder: You are right. In a paper by Lindsey Smith and myself (1998), we noticed with some surprise that the WN stars without hydrogen cannot just be accounted by one parameter, i.e., the mass, as we thought they would do. Thus, we considered rotation as one of the possibility to account for this second parameter. At the present stage, we have not enough calculations ready to check this point.

Heap: Would you tell us your definition of a WR star: is it defined by surface composition and/or by mass-loss rate?

Maeder: Your question addresses to the general problem of the correspondence between models and spectral classification, which is necessarily treated in a too schematic way, until we do reproduce the complete spectrum of the models. We consider that a star is a WR star when the $\mathrm{H}$-content by mass, $\mathrm{X}$, is lower than 0.4 in the corresponding range of luminosities. Would we take 0.3 or 0.45 , the results would not be too different. 